\title{
Effects of allopurinol and steroids on inflammation and oxidative tissue damage in experimental lens induced uveitis: a biochemical and morphological study
}

A J Augustin, M Spitznas, W Sekundo, F Koch, J Lutz, D Meller, F H Grus, A Wegener, S H-H Blumenröder

\begin{abstract}
Aims-To evaluate the effects of allopurinol in lens induced uveitis (LIU) by morphological methods and to compare these effects with those of steroids and a combination of both drugs biochemically and morphologically.
\end{abstract}

Methods-Lipid peroxides (LPO) of the retinal tissue were determined by two different methods (thiobarbituric acid assay (TBA) and high performance liquid chromatography expressed as malondialdehyde-like substances). Myeloperoxidase (MPO) activity in the iris/ciliary body complex was analysed spectrophotometrically. Histological changes on three morphological levels of LIU eyes were evaluated.

Results-Both allopurinol and the combination of allopurinol/prednisolone led to a significant reduction in the increased retinal LPO values. Prednisolone only revealed significant effects on retinal LPO when being measured with the TBA method. MPO activity in iris and ciliary body was significantly reduced in all therapy groups. The morphological evaluation of the sections by two masked investigators revealed a significant reduction $(p<0.05)$ in the inflammation score in all therapy groups. Morphometric studies using the QUANTIMED system (Leica, Cambridge) showed significantly reduced values $(p<0.05)$ in the allopurinol group and in the group receiving prednisolone and allopurinol. Prednisolone alone did not lead to a significant reduction in the values. Conclusions-The findings show that both allopurinol and steroids exert positive effects on the variables determined in LIU. The effects of steroids are believed to be mostly due to their direct action on inflammatory cells. The recently reported scavenging effects of methylprednisolone should play a minor role in this disease model. Allopurinol and oxypurinol act as direct scavengers of free radicals and hypochlorous acid, which is produced via MPO catalysis, thus leading to a reduction in tissue inflammation and tissue damage. (Br f Ophthalmol 1996; 80: 451-457)

In direct relation to the dose administered allopurinol was shown to countercheck the oxidative tissue damage following lens induced uveitis (LIU). ${ }^{1}$ This is believed to be due to its free radical scavenging activity and the scavenging of hypochlorous acid by its major metabolite oxypurinol. On the other hand, steroids applied as standard therapy for uveitis are known to reduce the synthesis of prostaglandins and thromboxanes by inhibition of the liberation of arachidonic acid from phospholipids. Moreover, several steroids such as methylprednisolone have scavenger activities that are dose dependent. ${ }^{23}$

In the present study, biochemical measurements were performed to compare the therapeutic effects of allopurinol with those of steroids under the same disease conditions. In order to evaluate whether steroids and allopurinol show additive therapeutic effects the two drugs were given together.

Additionally, the therapeutic effects were analysed histologically, using two methods.

\section{Materials and methods}

\section{ANIMAL EXPERIMENTS}

The treatment of animals conformed to the ARVO resolution on the use of animals in research. The experiments were performed with male Wistar rats $(n=37)$. The animals were divided randomly into seven groups (two groups, five animals each for the biochemical study; five groups, five or six animals each for the slit-lamp/histological study). The values of the basic tissue levels, controls and the values of the allopurinol group (biochemical analysis only) were obtained from previous studies. ${ }^{1}$ These values are established laboratory standards. The animals were anaesthetised using ether and the tissue samples were taken as described below.

Experimental uveitis was induced according to Rao et al. ${ }^{4}$ The animals were sensitised every 2 weeks over a 2 month period with four subcutaneous injections of bovine lens protein $10 \mathrm{mg}$ in complete Freund's adjuvant (Sigma, Deisenhofen, Germany). One week after the last injection the lens of one eye was ruptured using the tip of a 30 gauge needle. At the time of lens rupture the therapy groups were given allopurinol $(50 \mathrm{mg} / \mathrm{kg}$ body weight; Henning, Berlin, Germany), methylprednisolone $(7.5 \mathrm{mg} / \mathrm{kg}$ body weight; Hoechst, Frankfurt, Germany), or a combination of the two, intravenously. 


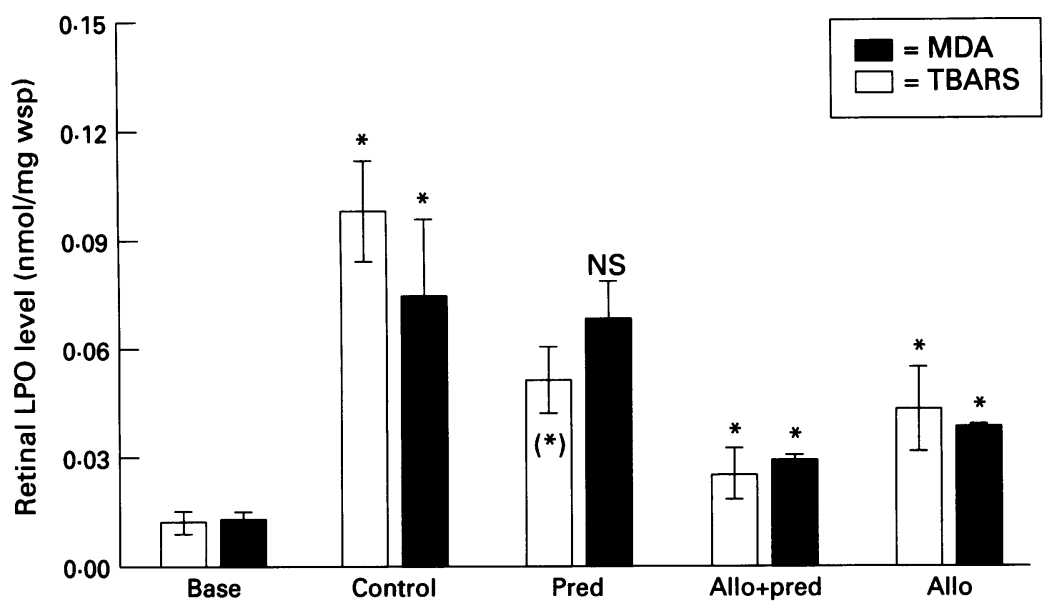

Figure 1 Lipid peroxide (LPO) levels in retinal tissue as base values (base), untreated lens induced uveitis (control) and after application of allopurinol (50 $\mathrm{mg} / \mathrm{kg}$ body weight), methylprednisolone $(7.5 \mathrm{mg} / \mathrm{kg}$ body weight $)$, and a combination of the two drugs. Untreated uveitis controls are statistically compared with base levels. All treatment values are statistically compared with untreated uveitis controls. Methylprednisolone showed a significant difference from untreated controls with the thiobarbituric acid method only $\left({ }^{\star} p<0.05\right)$. samples are expressed as means of $\mathrm{MDA} / \mathrm{mg}$ water soluble protein (SEM). MDA values of the aqueous humour are expressed as means $/ \mathrm{ml}$.

Thiobarbituric acid reactive substances (TBARS) Tissue samples were homogenised in $0.9 \mathrm{ml}$ $1.15 \% \mathrm{KCl}$ for 30 seconds with an Ultra Turrax blender and centrifuged at $3000 \mathrm{~g}$ for 5 minutes. The assay mixture consisted of $0 \cdot 1 \mathrm{ml}$ of the supernatant, $2 \mathrm{ml}$ of $0.9 \% \mathrm{NaCl}, 0.2 \mathrm{ml}$ of sodium dodecylsulphate, and $3 \mathrm{ml}$ of TBA, containing equal parts of $0.8 \%$ aqueous TBA and acetic acid. TBARS values are reported as means of TBARS/mg water soluble protein (SEM). ${ }^{8}$

\section{Myeloperoxidase (MPO)}

MPO activity in the iris and ciliary body was determined using the method of Bradley et al. ${ }^{9}$ To free MPO from primary granules of neutrophilic leucocytes, specimens were homogenised with an Ultra Turrax blender for 5 seconds in $3 \mathrm{ml}$ of hexadecyl trimethylammonium bromide (HTBA) solution $(0.5 \%$ HTBA in $50 \mathrm{mM}$ phosphate buffer, $\mathrm{pH} 6.0$ ). The homogenate was sonicated for 10 seconds, freeze thawed three times, and centrifuged at $40000 \mathrm{~g}$ for 15 minutes at $4^{\circ} \mathrm{C}$, resulting in the formation of a stable pellet. The supernatant was assayed for MPO activity by spectrophotometry using a mixture of $0.1 \mathrm{ml}$ of supernatant and $2.9 \mathrm{ml}$ of $50 \mathrm{mM}$ phosphate buffer, pH 6.0 , containing $0.167 \mathrm{mg} / \mathrm{ml}$ of $o$-dianisidine hydrochloride and $0.0005 \%$ hydrogen peroxide. The change in light absorbance at $460 \mathrm{~nm}$ was measured with a spectrophotometer. MPO values are expressed as $\mathrm{U} / \mathrm{mg}$ water soluble protein (SEM). ${ }^{8}$ according to the method of Esterbauer et al. ${ }^{5}$ Determination of LPO with the thiobarbituric acid (TBA) assay, expressed as TBA reactive substances (TBARS), was performed according to Ohkawa $e t \mathrm{al}^{6}{ }^{6}$ as modified by Augustin and Lutz. ${ }^{7}$

Both methods (MDA, TBARS) were compared and a correlation was calculated.

\section{Malondialdehyde-like substances (MDA)}

HPLC was performed using an instrument by Bio-Rad (Munich, Germany). The retina sample was suspended in acetonitrile, homogenised using an Ultra Turrax blender (Janke \& Kunkel, Staufen, Germany) and centrifuged at $3000 \mathrm{~g}$ for 5 minutes.

Clear supernatant $20 \mu \mathrm{l}$ was injected into the HPLC apparatus. The following columns were used for direct determination of MDA: (a) $250 \times 4.5 \mathrm{~mm} \quad \mathrm{~S} 5 \quad \mathrm{NH}_{2}$ spherisorb (aminophase); (b) precolumn $50 \times 4.6 \mathrm{~mm}$ (aminophase) (Promochem, Wesel, Germany). The upper pressure limit of the high pressure pump was set at 5000 psi. An injection valve with a $20 \mu \mathrm{l}$ loop and a $50 \mu \mathrm{l}$ syringe were used. The ultraviolet detector was set at $270 \mathrm{~nm}$. Values were calculated automatically with the aid of a computer and correlated with the protein content of the retinal sample, water soluble protein $/ \mathrm{ml}$. MDA values of the retina
HISTOLOGICAL STUDY

For the histological study the animals were deeply anaesthesised using ether. The lateral

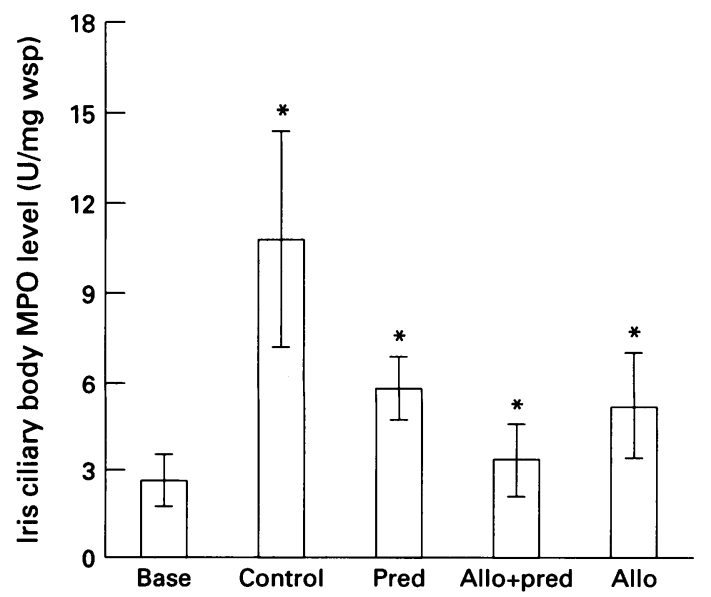

Figure 2 Myeloperoxidase (MPO) activity in iris and ciliary as base values (base), untreated lens induced uveitis (control) and after application of allopurinol $(50 \mathrm{mg} / \mathrm{kg}$ body weight), methylprednisolone ( $7.5 \mathrm{mg} / \mathrm{kg}$ body weight), and a combination of the two drugs. Untreated uveitis controls are statistically compared with base levels. untreated uveitis controls $\left({ }^{\star} p<0.05\right)$. All treatment values are statistically compared with 
Table 1 Scoring and QUANTIMED evaluation of cyclitis, iritis, and calculated uveitis values (means (SD)). The therapy values are compared with untreated controls

\begin{tabular}{|c|c|c|c|c|c|c|c|c|c|c|c|c|}
\hline & \multicolumn{3}{|l|}{ Control } & \multicolumn{3}{|l|}{ Allopurinol } & \multicolumn{3}{|c|}{ Methylprednisolone } & \multicolumn{3}{|l|}{ Combination } \\
\hline & Cyclitis & Iritis & Uveitis & Cyclitis & Iritis & Uveitis & Cyclitis & Iritis & Uveitis & Cyclitis & Iritis & Uveitis \\
\hline $\begin{array}{l}\text { Scoring } \\
\text { QUANTIMED }\end{array}$ & $\begin{array}{l}2 \cdot 97(0 \cdot 74) \\
644(128)\end{array}$ & $\begin{array}{l}3 \cdot 11(0 \cdot 84) \\
444(190)\end{array}$ & $\begin{array}{l}3.04(0 \cdot 77) \\
544(151)\end{array}$ & $\begin{array}{c}1.75(0.80)^{\star} \\
461(173) \\
(\mathrm{NS})\end{array}$ & $\begin{array}{l}2.02(0.80)^{\star} \\
208(77)^{\star}\end{array}$ & $\begin{array}{l}1.89(0.66)^{\star} \\
334(117)^{\star}\end{array}$ & $\begin{array}{c}1.83(0.50)^{\star} \\
626(55) \\
(\mathrm{NS})\end{array}$ & $\begin{array}{c}1.88(0.58)^{\star} \\
258(78) \\
(N S)\end{array}$ & $\begin{array}{c}1.86(0.52)^{\star} \\
442(62) \\
(\mathrm{NS})\end{array}$ & $\begin{array}{c}1.56(0.39)^{\star} \\
435(180) \\
(\text { NS) }\end{array}$ & $\begin{array}{l}1.79(0.45)^{\star} \\
175(67)^{\star}\end{array}$ & $\begin{array}{l}1.67(0.4)^{\star} \\
314(100)^{\star}\end{array}$ \\
\hline
\end{tabular}

${ }^{\star} \mathrm{p}<0 \cdot 05 . \mathrm{NS}=$ not significant.

aspects of the globes were marked by suture. Immediately thereafter a non-traumatic enucleation was performed and the animals were killed by exsanguination. The eyes were fixed by immersion in cold Karnovsky's fixative (4\% paraformaldehyde, $2.5 \%$ glutaraldehyde buffered in $0 \cdot 1 \mathrm{M} \mathrm{Na} \mathrm{HPO}_{4}$, and $0 \cdot 1 \mathrm{M}$ $\left.\mathrm{KH}_{2} \mathrm{PO}_{4}\right){ }^{10}$ Numbers were randomly assigned to the enucleated globes by a masked technician. Then the globes were divided in the horizontal plane. One half of the specimen was embedded in paraffin. Sections of $4 \mu \mathrm{m}$ were cut on a sliding microtome. In total, three levels (at the distance of 20 and 10 serial section, respectively) were prepared. The

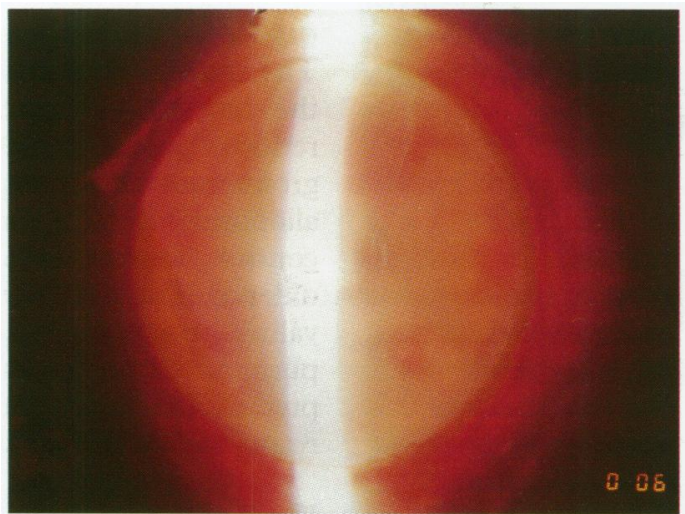

Fig $3 A$

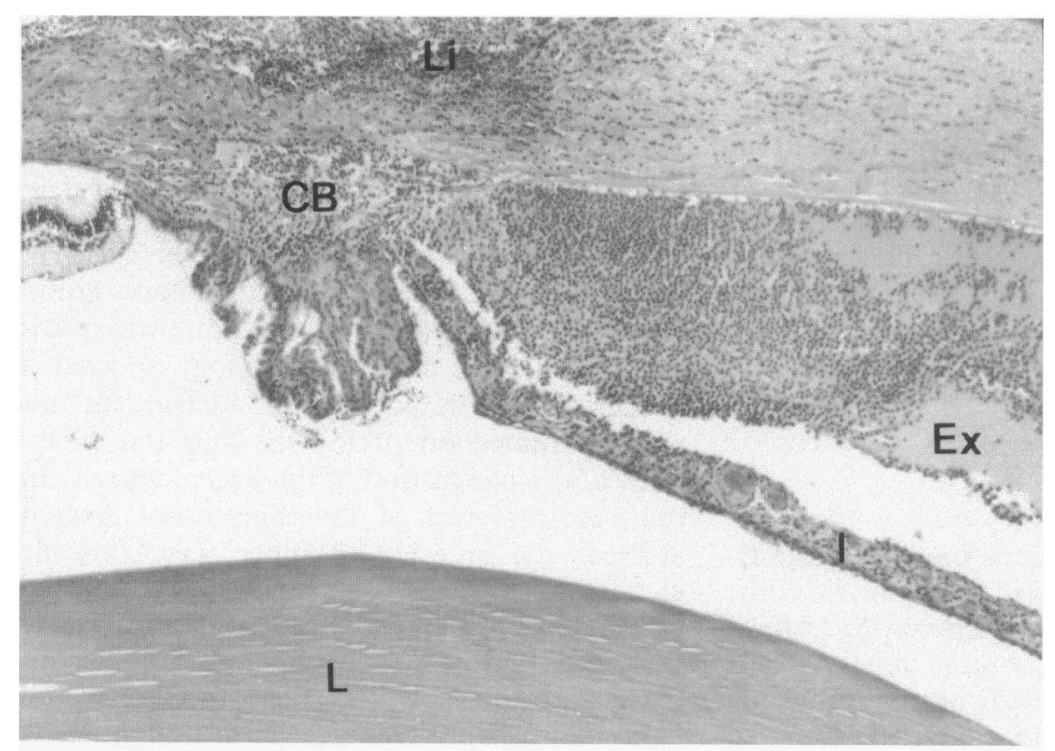

Fig $3 B$

Figure 3 Control group. (A) Slit-lamp photograph shows a diffuse haze in the anterior chamber obscuring the entire iris structure. (B) Histologically, there is gross inflammatory infiltration of the limbus (limbitis $=L i$ ) and of the peripheral cornea. The ciliary body $(C B)$ and the iris (I) also exhibit distinct infiltration with round cells. The anterior chamber is filled with an eosinophilic proteinaceous exudate $(E x)$, lymphocytes, and leucocytes. Note congested iris vessels, which correlate with inflammatory induced hyperaemia. $L=$ crystalline lens. (Haematoxylin and eosin, magnification $\times 75$.) sections were stained with haematoxylin and eosin.

Two independent, masked investigators (DM, WS) examined the numbered sections using a $1+$ to $4+$ score, depending on the degree of intraocular inflammation, which was morphologically seen as an accumulation of lymphocytes and polymorphonuclear granulocytes. The evaluation was done separately for iris and ciliary body tissue in the described manner. Thereafter, the histological numbers were reassigned to the original therapeutic groups.

In an independent, parallel setting a total area of the cell nucleus within a standardised region of the iris and the ciliary body of each level was calculated in a standardised manner using a modified QUANTIMED 500C imaging analysis system (Leica, Cambridge) connected to an Olympus light microscope (IMT-2, Olympus Optical Co (Europa) $\mathrm{GmbH}$, Hamburg, Germany).

With the investigator scores as well as with the QUANTIMED scores a combined uveitis score was calculated from the average of the iritis and the cyclitis scores in each sample.

\section{BIOMICROSCOPY}

Before enucleation, both eyes of each animal were inspected with a slit-lamp microscope (Zeiss photo slit-lamp microscope) and exemplary cases were photographed on Agfachrome RS 100 colour slide film. For this inspection, dilatation of the pupils was not attempted, because inflammation induced miosis was present in all LIU eyes. All optical examinations were performed in a dark room.

\section{STATISTICAL ANALYSIS}

\section{Biochemical analysis}

Statistical analysis of MDA/TBARS values in retinal tissue samples and MPO values was performed by Student's $t$ test. Normal distribution was assumed.

Tissue evaluation by scoring and by the QUANTIMED system

For each type of tissue investigated (iris and ciliary body), a separate statistical analysis of the scores was performed. The average of the histological iritis score and the cyclitis score was calculated as the combined uveitis score. This calculation was applied both to the quantitative results of the QUANTIMED system and to the scores determined by the two masked investigators. To determine the 


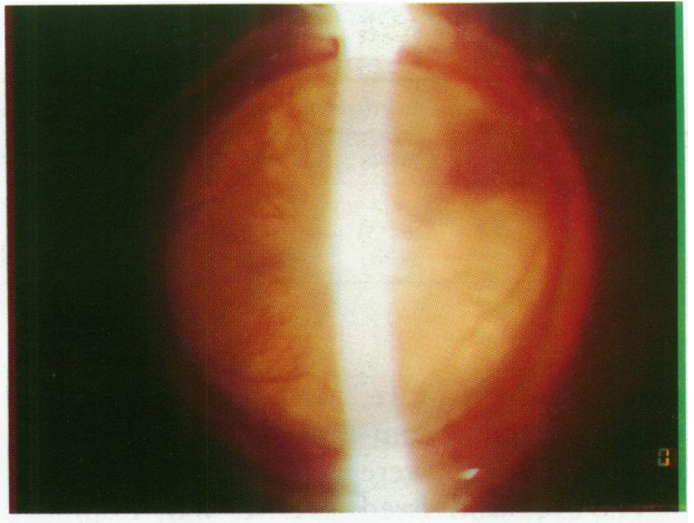

Fig $4 A$

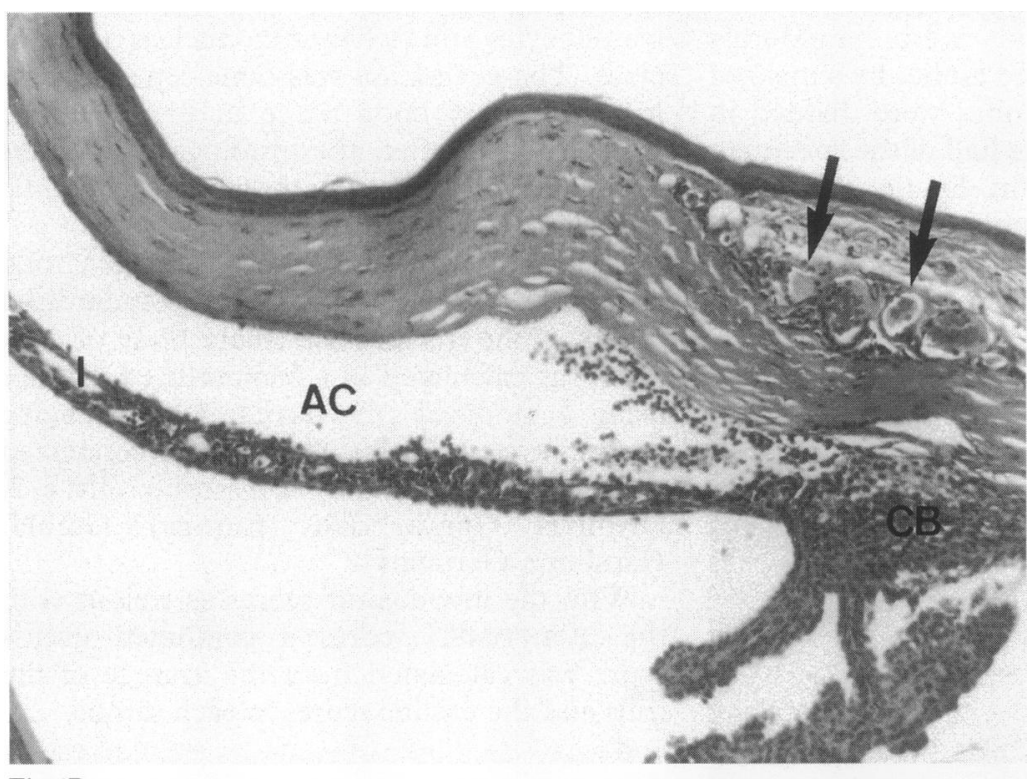

Fig $4 B$

Figure 4 Methylprednisolone group. (A) Slit-lamp photograph shows a moderate haze. However, the small iris vessels are not discernible. (B) Histologically, there is virtually no limbitis, but a distinct congestion of limbal vessels (arrows). The ciliary body (CB) and the iris (I) exhibit moderate inflammatory infiltration. Only a few scattered inflammatory cells are seen in the chamber angle. AC=anterior chamber. Compare with Figure 3.

(Haematoxylin and eosin, magnification $\times 75$.)

difference between groups, the MannWhitney-Wilcoxon test (U test) included in standard statistical software (SPCC-PC, SPSS Chicago, IL, USA) was used. The exact limit values of the $U$ test were used. The $p$ values given in the text are two sided values of this test.

\section{Results}

BIOCHEMICAL STUDY

Retinal lipid peroxide values increased significantly in untreated uveitis controls as compared with base values $(p<0.05$; values obtained from Augustin et $a l^{1}$ ). A significant decrease in comparison with untreated uveitis controls was achieved with a dose of $50 \mathrm{mg} / \mathrm{kg}$ body weight of allopurinol $(p<0.05$; values obtained from Augustin et $a l^{1}$ ).

When given alone, prednisolone resulted in a slight, not significant reduction in the values in comparison with those obtained by the HPLC method. However, a significant reduction $(p<0.05)$ was achieved when the results obtained with the TBA method were compared with each other.

A combination of the two drugs resulted in a significant reduction in the LPO values (Fig 1). The correlation of the two methods was 0.897 .

Iris and ciliary body showed a significant increase in myeloperoxidase activity $(p<0.05)$ as compared with base values (values obtained from Augustin et $a l^{1}$ ). A significant reduction in MPO ( $p<0.05)$ was achieved using allopurinol (values obtained from Augustin et $a l^{1}$ ), prednisolone, and a combination of the two drugs. The combination of allopurinol and prednisolone did not produce any additional significant changes compared with what were obtained by the single administration of each substance (Fig 2).

\section{MORPHOLOGICAL STUDY (TABLE 1)}

Scoring by two investigators

In all therapy groups the tissue inflammation (uveitis) was significantly reduced as compared with untreated controls $(p<0 \cdot 05)$.

\section{QUANTIMED evaluation}

The tissue inflammation (uveitis) evaluated by the QUANTIMED system revealed significantly reduced values $(p<0.05)$ in the allopurinol group and the group receiving steroid and allopurinol as compared with untreated controls. In the present study, the steroid alone did not lead to a significant reduction in the values $(p=0 \cdot 18)$. The therapy groups (allopurinol, prednisolone, and allopurinol/ prednisolone) did not differ significantly with respect to the uveitis, iritis, or cyclitis scores.

Figures 3 to 6 show representative slit-lamp photographs of the uveitis eye (A) and the corresponding microphotograph of a histological section (B) of each study group. In the control group anterior chamber structures were barely seen, because of an extensive flare (Fig 3A). On histology the flare correlated with a massive proteinaceous exudate that is rich in inflammatory cells derived from grossly inflamed anterior chamber structures (Fig 3B). In contrast, in the methylprednisolone group the slit-lamp view of the iris structure was much better (Fig 4A) and the histology showed a moderate, mainly polymorphonuclear, inflammatory infiltration of the iris and the ciliary body and a congestion of the limbal vessels. In the vast majority of the allupurinol treated animals the anterior chamber was clinically clear (Fig 5A). This observation was confirmed by microscopy. Here, only minor sequelae of the severe Arthus reaction, as shown in the control group, could be seen (Fig 5B). Clinical and light microscopic changes in the allopurinol/ methylprednisolone group were, to a certain extent, comparable with those seen in the allopurinol treated animals (Figs $6 \mathrm{~A}$ and $6 \mathrm{~B}$ ).

\section{Discussion}

The morphological results confirm previously 


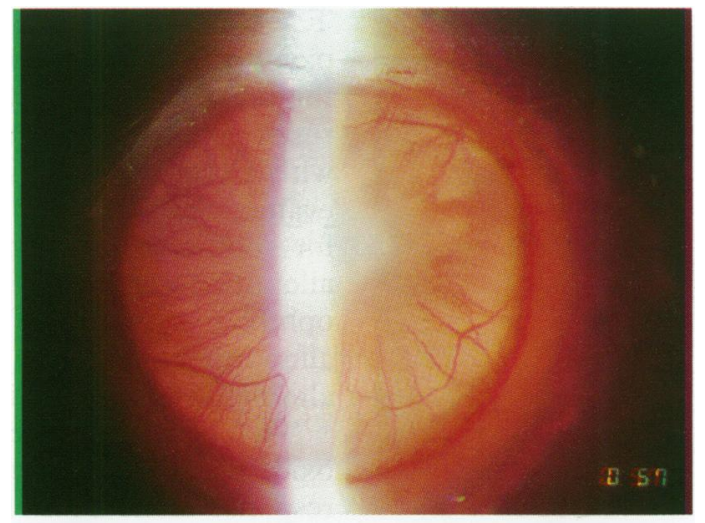

Fig 5 A

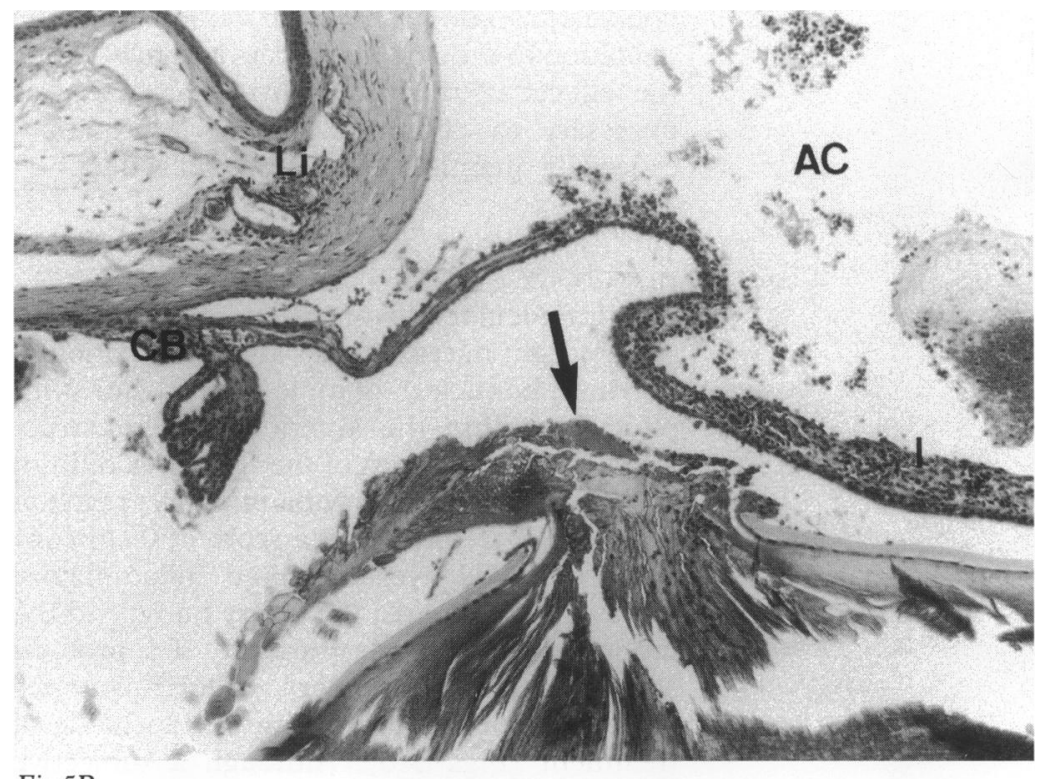

Fig $5 B$

Figure 5 Allopurinol group. (A) Slit-lamp photograph clearly shows details of the iris vasculature. (B) The limbus (Li) and the ciliary body (CB) are almost free of inflammatory cells. The latter are scattered through the iris stroma (I) and to a minor extent within the anterior chamber $(A C)$. Inflammatory cells are also seen adjacent to the site of the anterior lens capsule rupture, where the lens cortex is protruding forward (arrow). Compare with Figure 3. (Haematoxylin and eosin, magnification $\times 75$.)

reported biochemical data regarding the beneficial effects of allopurinol on the inflammatory processes in LIU. ${ }^{1}$ The effects of allopurinol at a dose of $50 \mathrm{mg} / \mathrm{kg}$ body weight were comparable with those of steroids when given at a dose of $7.5 \mathrm{mg} / \mathrm{kg}$ body weight using the scoring evaluation. A combination of the two drugs did not show significant additive effects either biochemically or morphologically.

\section{BIOCHEMICAL RESULTS}

In the present study the variables of inflammation (MPO) and oxidative tissue damage (LPO) of the affected ocular structures were evaluated systematically.

Inflammation and inflammation associated reactions are believed to be the major source of free radicals leading to oxidative tissue damage following uveitis. ${ }^{411-14}$

In the present study the oxidative tissue damage was evaluated by two methods. The great advantage of the thiobarbituric acid method is its high sensitivity in detecting peroxides. However, the main disadvantage of this method is its lower specificity. Therefore, the two methods were used together and a correlation was calculated. The problem of just using the TBA method is documented in the results of the steroid group. In particular, values such as the steroid values bordering the threshold of significance may give different results with each of the two methods. One reason for this difference might be the higher sensitivity of the TBA method, which may lead to a higher variation of the values. Thus, it is conceivable that the steroid results can be regarded as a less pronounced improvement when compared with allopurinol and/or combination therapy. This fact is also documented in the morphological data, which demonstrate a significant improvement when using the score system and no significant improvement when using the QUANTIMED evaluation.

\section{Methylprednisolone}

Steroids influence several mechanisms leading to oxidative metabolites and reduce the accumulation of neutrophils and macrophages in the inflamed tissue. ${ }^{11}{ }^{15-18}$ Steroids do not prevent the synthesis of antibodies. Their immunological effects are based on the reduction of the inflammatory response to immunological processes and the prevention of the passage of antigen-antibody complexes through basal membranes. Apart from the reduction of the absolute number of phagocytic cells in the inflamed tissue steroids influence the arachidonic acid cascade that might be one source of oxidative metabolites in the LIU model.

However, there are additional sources of oxidative reactions in activated inflammatory cells, such as the MPO derived production of hypochlorous acid and the respiratory burst leading to powerful radicals such as the hydroxyl radical and a singlet oxygen, both being capable of tissue oxygenation. ${ }^{4}$ One might assume that the antioxidative effects of steroids are only the result of a reduction in the absolute number of inflammatory cells and the specific action on different pathways capable of producing oxidative metabolites. Another explanation for the minor effects of steroids when compared with those obtained by powerful scavengers such as allopurinol and/or oxypurinol, which are able to influence inflammatory induced free radicals and radicals from other sources, is offered by the recently reported free radical scavenging activity of methylprednisolone..$^{23}$ Using a model of spinal cord injury this study showed that the corticosteroid methylprednisolone possesses significant antioxidant efficacy when administered in doses exceeding those given in the present study. ${ }^{23}$

The free radical scavenging activity of methylprednisolone is in no way related to the steroid's glucocorticoid receptor mediated actions. ${ }^{2} 3$ Structurally similar drugs such as lazaroids are also known to be both potent scavengers of hydroxyl radical/superoxide anion radicals and inhibitors of activated phagocytic cells. ${ }^{19}$ 


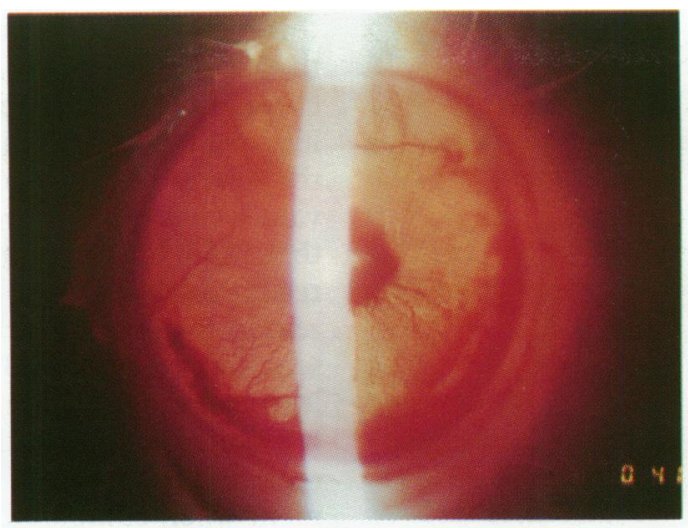

Fig $6 A$

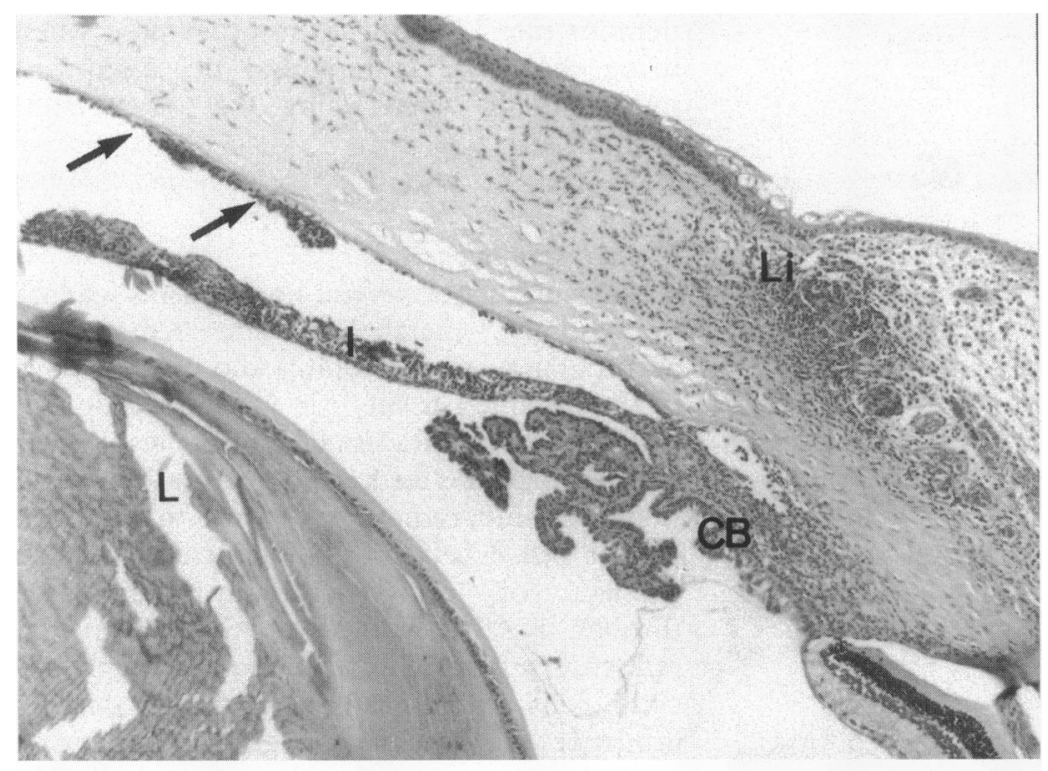

Fig $6 B$

Figure 6 Allopurinol/methylprednisolone group. (A) Slit-lamp photograph shows a fairly clear view of the anterior chamber structures similar to Figure 5A. An incidental hyphaema is due to a paracentesis at the time of lens rupture. (B) While there is moderate limbitis ( $\mathrm{Li}$ ), the iris (I) and ciliary body (CB) exhibit barely discernible signs of inflammation. A few inflammatory precipitates are adjacent to the corneal endothelium (arrows). $L=$ crystalline lens. Compare with Figure 3. (Haematoxylin and eosin, magnification $\times 75$ ).

Thus, it is conceivable that when given in doses below the scavenger dose of $30 \mathrm{mg} / \mathrm{kg}$ body weight methylprednisolone acts only on inflammation and thus only prevents the oxidative damage originating directly from the inflammation. The radicals already being generated and those originating from other sources such as iron are not influenced because the steroid dose is too low. These facts are confirmed by our results, which showed a significant reduction in MPO activity, but less pronounced effects on lipid peroxides in the retinal tissue.

The aim of this study was to compare the action of allopurinol with steroids at a dose range given in uveitis therapy. The doses necessary for scavenging activities will not be given in ophthalmology. Nevertheless, the LIU model might be suitable for a pharmacodynamic study of the scavenging effects of steroids.

\section{Allopurinol und oxypurinol}

In a previous study ${ }^{1}$ we demonstrated that allopurinol reaches the ocular tissues at levels necessary for a scavenger activity of this drug.
The scavenging effects of both allopurinol and oxypurinol in LIU were discussed in detail. In the present study we confirm the biochemical results achieved by morphological evaluation with a score system and with the QUANTIMED evaluation. Our findings indicate the necessity for additional investigations on the effects of allopurinol and oxypurinol on inflammatory ophthalmic diseases. The topical application of allopurinol particularly shows promise as an alternative to steroids in several diseases.

The beneficial effects of these drugs on oxidative tissue damage and inflammatory response alone do not provide enough information regarding the beneficial effects on the whole disease process. Therefore, to draw conclusions about the contribution of tissue oxidation to the disease process and to evaluate the effects of the drugs investigated, it was necessary to obtain additional information, especially quantitative morphological data.

\section{HISTOLOGY}

The intraocular inflammation could easily be seen by light microscopy as an accumulation of polymorphonuclear granulocytes and/or lymphocytes within the anterior segment structures. The fellow eyes of the LIU eyes did not show any significant inflammatory reaction and were therefore given a score of 0 . In contrast, the LIU eyes exhibited inflammatory infiltration of the iris, the ciliary body, and the limbus, as well as the presence of a proteinaceous and inflammatory cellular anterior chamber exudate, depending on the mode of treatment (Figs 3-6). Although a subjective impression cannot be entirely ruled out in any morphological study, we attempted to minimise this influence not only by randomisation of the enucleated globes, but also by (1) masking the investigators, (2) contributing examinations at three different levels, and (3) defining strict score criteria, ranging from 0 in eyes with no inflammation to $4+$ in eyes in which the above mentioned structures exhibited pronounced inflammatory alterations. Indeed, after reassignment, only the untreated eyes yielded the maximum score. The main advantage of the morphological method is that it examines the entire globe using different magnifications, delivering a comprehensive impression. It is not surprising that the treated eyes contained fewer inflammatory cells than untreated ones.

The morphometric studies using the modified image analyser QUANTIMED were performed in an attempt to gain better objectivity of the histological data. Again, the main advantage of this system is an investigator independent calculation of the nuclear area, as defined by the basophilic colour of the nuclei on haematoxylin and eosin stained sections. However, the disadvantages of the method were as follows: (1) the calculations were carried out on a defined area of the iris and the ciliary body irrespective of the degree of the inflammatory changes. Thus, this selection did not always contain a representative area; $(2)$ in particular, both the ciliary body and the iris 
possess epithelial and stromal cells with basophilic nuclei whose area was also calculated. Although we assumed these values to be equal in all eyes, there are probably differences between individual eyes. This could be one explanation as to why the prednisolone treated group did not show a significant effect with regard to anterior uveitis and cyclitis alone, but was significant in the iritis group. In fact, the cellular density of the ciliary body is much higher than that of the iris. Therefore, the inflammatory cell infiltration of the iris stroma calculated as an area value is not as high in absolute numbers as is the infiltration of the ciliary body.

Supported by Deutsche Forschungsgemeinschaft (DFG), AU 126/1-1

1 Augustin AJ, Böker T, Blumenröder S H-H, Lutz J, Spitznas $M$. Free radical scavenging and antioxidant activity of allopurinol and oxypurinol in experimental lens-induced uveitis. Invest Ophthalmol Vis Sci 1994; 35: 3897-904.

2 Hall ED. Lipid antioxidants in acute central nervous system injury. Ann Emerg Med 1993; 22: 1022-7.

3 Hall ED. The effects of glucocorticoid and nonglucocorticoid steroids on acute neuronal degeneration. Adv Neurol coid steroids on

4 Rao NA, Calandra AJ, Sevanian A, Bowe B, Delmage JM, Marak GE. Modulation of lens induced uveitis by superoxide dismutase. Ophthalmic Res 1986; 18: 41-6.

5 Esterbauer H, Lang J, Zadravec S, Slater TF. Detection of malonaldehyde by high-performance liquid chromatography. Methods Enzymol 1984; 105: 319-28.

6 Ohkawa H, Ohishi N, Yagi K. Assay for lipid peroxides in animal tissues by thiobarbituric acid. Anal Biochem 1979; 95: 351-8.
7 Augustin AJ, Lutz J. Intestinal, hepatic and renal production of thiobarbituric acid reactive substances and myeloperoxidase activity after temporary aortic occlusion and reperfusion. Life Sci 1991; 49: 961-8.

8 Schacterle GR, Pollack RL. A simplified method for the quantitative assay of small amounts of protein in biologic material. Anal Biochem 1973; 51: 654-5.

9 Bradley PP, Priebat DA, Christensen RD, Rothstein G. Measurement of cutaneous inflammation: estimation of neutrophil content with an enzyme marker. $\mathcal{f}$ Invest neutrophil content with

10 Karnovsky M. A formaldehyde-glutaraldehyde fixative of high osmolarity for use in electron microscopy. $\mathcal{F}$ Cell Biol 1965; 27 : 137-8.

11 Bazan NG, Abreu MT de, Bazan HEP, Belfort $R$. Arachidonic acid cascade and platelet activating factor in the network of eye inflammatory mediators: therapeutic implications in uveitis. Int Ophthalmol 1990; 14: 335-44

12 Rao NA. Role of oxygen free radicals in retinal damage associated with experimental uveitis. Trans Am Ophthalmol Soc 1990; 88: 797-850.

13 Wu GS, Sevanian A, Rao NA. Detection of retinal lipid u GS, Sevanian A, Rao NA. Detection of retinal lipid hydroperoxides in

14 Goto $\mathrm{H}$, Wu GS, Gritz DC, Atalla LR, Rao NA. Chemotactic activity of the peroxidized retinal membrane lipids in experimental autoimmune uveitis. Curr Eye Res 1991; 10: 1009-14.

15 Weissmann G, Sessa G, Bevans V. Effect of DMSO on the stabilization of lysosomes by cortisone and chloroquine in vitro. Ann NY Acad Sci 1967; 141: 326-32.

16 Gadelata D, Verma M, Davis JM. Inhibition of neutrophil leukotriene generation by the 21 aminosteroid, Uleukotriene generation by the 21

17 Borgeat P, Samuelsson B. Transformation of arachidonic acid by rabbit polymorphonuclear leukocytes. $\mathfrak{f}$ Biol Chem 1979; 254: 2643-6.

18 Ward PA. The chemosuppression of chemotaxis. $\mathcal{F}$ Exp Med 1966; 124: 209-26.

19 Thomas PD, Mao GD, Rabinovitch A, Poznanzky MJ. Inhibition of superoxide-generating NADPH oxidase of human neutrophils by lazaroids (21-aminosteroids and 2methylaminochromans). Biochem Pharmacol 1993; 45: 241-51. 